\title{
Secondary Discitis Masquerading as Treatment Failure of Primary Discitis: Case Report and Review of the Literature
}

\author{
GENNADIY A. KATSEVMAN, MD, ${ }^{1}$ ERIC EMERY BS, ${ }^{2}$ JOHN C. FRANCE, MD, ${ }^{3}$ CARA L. SEDNEY, MD, \\ $\mathrm{MA}^{1}$ \\ ${ }^{I}$ Department of Neurosurgery, West Virginia University, Morgantown, West Virginia, ${ }^{2}$ West Virginia University School of Medicine, Morgantown, West Virginia, \\ ${ }^{3}$ Department of Orthopaedics, West Virginia University, Morgantown, West Virginia
}

\begin{abstract}
Background: Vertebral osteomyelitis often presents with localized back pain at the site of infection and elevated inflammatory markers. It can generally be treated conservatively with antibiotics targeted at the causative microorganism, but failure of medical treatment often necessitates surgical debridement. There are no reports in the literature that describe a secondary infection masquerading as treatment failure of the primary infection.

Methods: We present a case of a 29-year-old male with a history of intravenous drug abuse who was treated with antibiotics for methicillin-sensitive Staphylococcus aureus T6-7 discitis/osteomyelitis. The patient later returned with worsened back pain and was initially thought to have experienced failure of medical therapy, but instead was found to have a secondary discitis at a different level, with biopsy-proven different microorganism (Enterobacter cloacae).

Conclusions: This case illustrates the possibility of secondary discitis masquerading as treatment failure of the primary discitis. Repeat imaging and biopsy of the new lesion avoided a surgical procedure in this patient.
\end{abstract}

Other \& Special Categories

Keywords: vertebral osteomyelitis, secondary discitis, treatment failure

\section{INTRODUCTION}

Vertebral osteomyelitis, also referred to as discitis osteomyelitis, septic discitis, or spondylodiscitis, represents $3-5 \%$ of all cases of osteomyelitis. ${ }^{1}$ Several etiologies are possible; vertebral osteomyelitis can be established via hematogenous spread in the setting of positive blood cultures, iatrogenically secondary to surgical procedures, directly as a result of trauma, or by contiguous spread from an adjacent infected source. A distant focus of infection has been identified in almost half of cases of osteomyelitis, with urinary tract infection being the most common source of infection., ${ }^{2,3}$ Other predisposing factors include diabetes, advanced age, intravenous (IV) drug use, immunosuppression, malignancy, renal failure, rheumatological disease, liver cirrhosis, previous spinal surgery, and coronary heart disease. ${ }^{2,4}$ The incidence of vertebral infections has been rising over time, thought to be due to increased detection rates, prevalence of IV drug use, health care-associated infections, spinal surgery, and an increase in the susceptible immunocompromised and aging populations. ${ }^{2}$ Intravenous drug users have higher frequency of multifocal involvement, which can be present in a continuous $(6 \%)$ or skipping $(3 \%)$ nature. $^{3}$

A microbiological diagnosis is essential to determine the ideal antimicrobial treatment regimen. Cultures from other foci of infection are important, as is biopsy of the affected vertebral region, preferably attained prior to antibiotic initiation. ${ }^{5}$ Overall, osteomyelitis is uncommon and can generally be treated conservatively with antibiotics, but has great potential for morbidity and mortality if not treated appropriately. Failure of medical treatment should be considered in patients with recurrent pain or rising inflammatory markers after treatment. In these cases, surgery is often required for debridement of the infection and possibly stabilization of the spine.

\section{CASE REPORT}

A 29-year-old male with a remote history of alcohol and IV drug abuse, morbid obesity, and hypertension presented with right-sided thoracic back pain for which he had previously undergone treatment for a presumed pneumonia. He exhibited leukocytosis with white blood cell (WBC) count of 


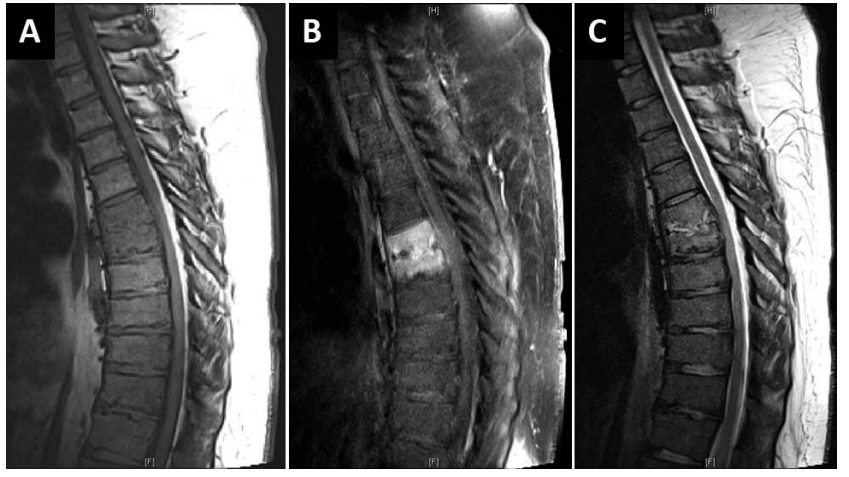

Figure 1. Magnetic resonance imaging of the thoracic spine demonstrates T6-7 discitis/osteomyelitis. (A) Sagittal T1-weighted and (B) T1-weighted with contrast images reveal abnormal enhancement at this level. (C) T2-weighted images demonstrate some endplate and disc edema at this level without significant cord compression.

$16900 / \mathrm{uL}$, as well as elevated C-reactive protein (CRP) of $23.8 \mathrm{mg} / \mathrm{L}$ and erythrocyte sedimentation rate (ESR) of $47 \mathrm{~mm} / \mathrm{h}$. Magnetic resonance imaging (MRI) was suggestive of T6-7 osteomyelitis and discitis (Figure 1). Multiple repeated blood cultures were positive for methicillin-sensitive Staphylococcus aureus (MSSA), although a transpedicular biopsy revealed no bacterial growth. Patient was started on 6 weeks of nafcillin from the day of first negative blood cultures per infectious disease recommendations. Leukocytosis resolved, and CRP trended down from the peak of 116.2 to $32.8 \mathrm{mg} / \mathrm{L}$. A peripherally inserted central catheter (PICC) was placed, and the patient was discharged with IV antibiotics with outpatient follow up. The patient stated he had not abused IV drugs for several years, so the medical teams involved deemed him safe for discharge home with a PICC line. The patient's pain was not significant enough to require an orthosis for added pain control.

The patient was followed clinically throughout his antibiotic course and demonstrated improved symptoms and laboratory values, as well as stable imaging. However, 2 weeks after ending his course of antibiotics, he again returned with back pain. Labs on presentation were WBC of $8200 / \mathrm{uL}$, CRP of $45.3 \mathrm{mg} / \mathrm{L}$, and $\mathrm{ESR}$ of $82 \mathrm{~mm} / \mathrm{h}$. Due to increasing back pain and worsening inflammatory markers after cessation of antibiotics, the patient was thought to have failed medical management of discitis with a tentative plan to take him to the operating suite for washout. On exam, however, he appeared to have pain in a lower location, near the thoracolumbar junction. As such, the MRI was repeated, which demonstrated interval development

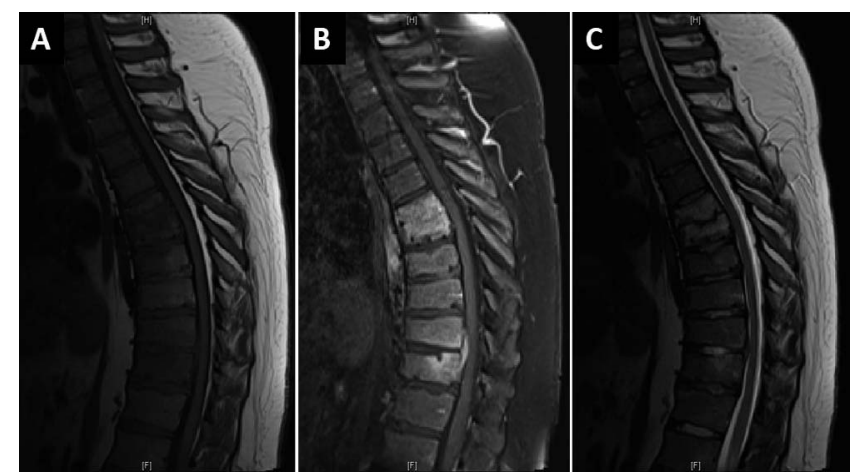

Figure 2. Magnetic resonance imaging (A) T1-weighted images without contrast, (B) with contrast, and (C) T2 sequences of the thoracic spine demonstrate interval development of T10-11 discitis/osteomyelitis. Redemonstrated is sequela of T6-7 discitis osteomyelitis.

of T10-11 discitis/osteomyelitis in addition to chronic findings of T6-7 discitis/osteomyelitis (Figure 2). No worsening of the T6-7 level was apparent. Repeat blood cultures were negative, and his prior PICC line had been previously removed after completion of antibiotics and was unable to be cultured. Patient underwent percutaneous fluoroscopically guided T11 transpedicular bone biopsy and T10-11 disc aspiration; cultures were positive for Enterobacter cloacae complex. Due to concern for previous PICC line misuse, patient completed an additional 6 weeks of IV cefepime antibiotic therapy as an inpatient to treat the Enterobacter infection with the belief that he responded appropriately and resolved the MSSA infection. Clinical and laboratory markers remained stable throughout, with no additional recurrence of symptoms after ending his second course of antibiotic therapy.

\section{DISCUSSION}

Diagnosis of vertebral osteomyelitis is based on clinical, laboratory, and radiological data and can often be missed or delayed given the insidious onset of symptoms and overall high prevalence of back pain in the general population. Magnetic resonance imaging provides the best sensitivity and specificity for detecting osteomyelitis, but other imaging modalities such as plain films, computed tomography (CT), or nuclear medicine imaging may be used in addition to or in place of MRI. ${ }^{6}$ Routine followup MRI imaging at 4 to 8 weeks after start of antibiotic therapy is generally not useful given that the imaging findings may lag the clinical response, and gadolinium enhancement at the site of infection 
may not resolve even after years of treatment. ${ }^{2}$ At times, the imaging may be misleading and may illustrate worsening of the infection despite an appropriate antibiotic response, decrease in inflammatory markers, and improvement in clinical symptoms. As such, current recommendations are against routine follow-up MRI imaging in patients who appear to be responding to antibiotics. $^{5}$ Furthermore, recommendations are against surgical debridement and/or stabilization in patients who have worsening bony imaging at 4-6 weeks in the setting of improvement in clinical symptoms, physical examination, and inflammatory markers. ${ }^{5}$ In patients who appear to fail treatment, additional imaging such as CT or MRI and further tissue sampling can be obtained. In our patient, the decision to obtain additional imaging centered on his pain being in a new location when compared to his previous discitis pain. The decision to biopsy this new area of infection was to avoid an extensive surgical procedure which would likely have to address both areas of discitis.

Intravenous drug abusers in particular represent a unique population of patients who may sustain vertebral osteomyelitis. They tend to be noncompliant with the treatment and follow up and often require inpatient hospitalization for the duration of the course of antibiotics. One study demonstrated that IV drug users tend to have a higher incidence of hardware failure than non-IV drug users. ${ }^{7}$ In a retrospective review of 102 cases of primary spine infection in patients with a history of IV drug use, the most common affected levels were lumbar spine $(57.8 \%)$, followed by thoracic $(23.5 \%)$, and cervical $(8.8 \%)$, similar to the distribution in non-IV drug use patients. ${ }^{8}$ A single level was involved in 71 patients $(69.6 \%)$, with the rest having involvement of multiple levels. Interestingly, $51 \%$ of patients had a normal WBC, $8.8 \%$ had a normal ESR, and 29\% had a normal CRP. The most common pathogens were MSSA (39.2\%) and MRSA (22.5\%), and 2 patients $(1.9 \%)$ required surgical intervention after failure of medical therapy which presented as increased back pain. In 1 review, $4 \%$ of patients had osteomyelitis in multiple vertebral levels, which was found to be more common in IV drug users. ${ }^{3}$ Continued drug use during treatment is a common issue in this setting and it is felt likely that the patient described in this case was actively abusing IV drugs as a cause for his primary and second infection. The decision to discharge him home for the first treatment cycle was made due to his assertion that he had not used in several years. The patient was kept inpatient for the duration of treatment at the second occurrence due to the likelihood of continued IV drug abuse.

One review demonstrated that relapse was encountered in $14 \%$ of cervical osteomyelitis patients with the main symptom being persistent pain. ${ }^{9}$ Another review demonstrated a relapse rate of $32 \% .^{3}$ In 1 series of 253 patients, the relapse rate was $14 \%$ with a range of $1-22 \%$ cited from the literature; for 29 of the 30 patients with cultureconfirmed relapse, the same microorganism was recovered during relapse as during the first episode of infection. ${ }^{10}$ In 1 patient with relapse caused by Staphylococcus aureus, Escherichia coli was isolated concomitantly with $S$. aureus from the infected intervertebral disc space, whereas only $S$. aureus was recovered from the same disc space during the first episode. There were no patients described as having different microorganisms in different segments of the spine. There is the possibility that this patient had a multiorganism infection with detection and treatment of only the MSSA initially, thus allowing aggressive growth of Enterobacter. Initial biopsy cultures were entirely negative with MSSA being discovered solely on blood cultures; it is possible that the biopsy was a false negative or that Enterobacter had simply not spread hematogenously at that time. However, it would be unusual for an Enterobacter discitis to respond clinically to nafcillin treatment alone. Multiple organism discitis has been presented previously. ${ }^{10}$

Treatment of vertebral osteomyelitis may consist of nonsurgical treatment with antibiotic therapyusually for a duration of 6 weeks - and possible immobilization with a brace. Hyperbaric oxygen therapy has also been described as adjuvant therapy in treatment of spinal osteomyelitis. ${ }^{11}$ Surgery is considered for patients who have failed medical treatment, have neurological deficits, concern of spinal instability or new deformity, require drainage of an abscess, and if tissue is desired for additional diagnostic support. Shah et al demonstrated 6 independent predictors of nonoperative failure in the setting of epidural abscess: a presenting motor deficit, pathologic or compression fracture in affected levels, active malignancy, diabetes mellitus, sensory changes, and dorsal location of abscess. ${ }^{12}$ Similar predictors likely exist in the setting of discitis/osteomyelitis. 
On occasion in the setting of spinal instability due to excessive infection or surgical debridement of infective focus, spinal stabilization with hardware is required and has not been shown to increase risk of recurrent infection. ${ }^{13-16}$ The surgical strategy should aim to provide neural decompression, spinal stabilization, and clearance of infection. ${ }^{17}$ In certain instances in persistent infection despite antibiotics, however, instrumentation removal is performed with the use of immobilization, external orthosis, or traction to manage instability if it is present.

Recurrence of osteomyelitis generally occurs with the same bacterial strain that failed to be eradicated initially and represents a failure of medical management, requiring surgery in many cases. However, we present a case report of a patient who represented with back pain after cessation of antibiotic therapy and was found to have a second episode of vertebral osteomyelitis at a separate site caused by a different strain of bacteria. This case illustrates the importance of considering infections at other locations as part of the differential diagnosis - in addition to failed medical treatment at the previous site of infection - when a patient returns with back pain. Particular location of back pain and symptomatology must be scrutinized. Repeat imaging such as MRI, although generally not indicated during the acute treatment of osteomyelitis, may be useful if an infection is suspected at a new site or if surgery is planned for the treatment of chronic osteomyelitis. Such repeat imaging has the potential of changing the treatment algorithm; a new focus of infection with a different pathogen obtained via biopsy can be attempted to be treated medically with appropriate antibiotics, and surgery may be avoided.

\section{CONCLUSIONS}

When a patient with a history of vertebral osteomyelitis returns with relapsed symptoms such as back pain, it is important to ascertain the location of the back pain and to determine whether it may be recurrent osteomyelitis at a new location. If so, additional imaging and a repeat biopsy may be warranted given that a different pathogen may be identified and further treated with appropriate antibiotics. This may be particularly relevant in those with IV drug abuse history.

\section{REFERENCES}

1. Sobottke R, Seifert H, Fatkenheuer G, Schmidt M, Gossmann A, Eysel P. Current diagnosis and treatment of spondylodiscitis. Dtsch Arztebl Int. 2008;105(10):181-187.

2. Gouliouris T, Aliyu SH, Brown NM. Spondylodiscitis: update on diagnosis and management. J Antimicrob Chemother. 2010;65(Suppl 3):iii11-iii24.

3. Mylona E, Samarkos M, Kakalou E, Fanourgiakis P, Skoutelis A. Pyogenic vertebral osteomyelitis: a systematic review of clinical characteristics. Semin Arthritis Rheum. 2009;39(1):10-17.

4. Zimmerli W. Clinical practice. Vertebral osteomyelitis. New Engl J Med. 2010;362(11):1022-1029.

5. Berbari EF, Kanj SS, Kowalski TJ, et al. 2015 Infectious Diseases Society of America (IDSA) clinical practice guidelines for the diagnosis and treatment of native vertebral osteomyelitis in adults. Clin Infect Dis. 2015;61(6):e26-e46.

6. Jevtic V. Vertebral infection. Eur Radiol. 2004;14(Suppl 3):E43-E52.

7. Wang Z, Lenehan B, Itshayek E, et al. Primary pyogenic infection of the spine in intravenous drug users: a prospective observational study. Spine (Phila Pa 1976). 2012;37(8):685692.

8. Ziu M, Dengler B, Cordell D, Bartanusz V. Diagnosis and management of primary pyogenic spinal infections in intravenous recreational drug users. Neurosurg Focus. 2014;37(2):E3.

9. Barnes B, Alexander JT, Branch CL Jr. Cervical osteomyelitis: a brief review. Neurosurg Focus. 2004;17(6):E11.

10. McHenry MC, Easley KA, Locker GA. Vertebral osteomyelitis: long-term outcome for 253 patients from 7 Cleveland-area hospitals. Clin Infect Dis. 2002;34(10):13421350 .

11. Ahmed R, Severson MA, Traynelis VC. Role of hyperbaric oxygen therapy in the treatment of bacterial spinal osteomyelitis. J Neurosurg Spine. 2009;10(1):16-20.

12. Shah AA, Ogink PT, Nelson SB, Harris MB, Schwab JH. Nonoperative management of spinal epidural abscess: development of a predictive algorithm for failure. J Bone Joint Surg Am. 2018;100(7):546-555.

13. Bydon M, De la Garza-Ramos R, Macki M, et al. Spinal instrumentation in patients with primary spinal infections does not lead to greater recurrent infection rates: an analysis of 118 cases. World Neurosurg. 2014;82(6):e807-e814.

14. Lee MC, Wang MY, Fessler RG, Liauw J, Kim DH. Instrumentation in patients with spinal infection. Neurosurg Focus. 2004;17(6):E7.

15. Rayes M, Colen CB, Bahgat DA, et al. Safety of instrumentation in patients with spinal infection. $J$ Neurosurg Spine. 2010;12(6):647-659.

16. Talia AJ, Wong ML, Lau HC, Kaye AH. Safety of instrumentation and fusion at the time of surgical debridement for spinal infection. J Clin Neurosci. 2015;22(7):1111-1116.

17. Hsieh PC, Wienecke RJ, O'Shaughnessy BA, Koski TR, Ondra SL. Surgical strategies for vertebral osteomyelitis and epidural abscess. Neurosurg Focus. 2004;17(6):E4.

\section{Disclosures and COI: None.}

Corresponding Author: Gennadiy A. Katsevman, MD, West Virginia University, Department of 
Neurosurgery, One Medical Center Drive, Suite 4300, Health Sciences Center, PO Box 9183, Morgantown, WV 26506-9183. Phone: (224) 4898384; Email: gkatsev@gmail.com.

Published 30 April 2019
This manuscript is generously published free of charge by ISASS, the International Society for the Advancement of Spine Surgery. Copyright (C) 2019 ISASS. To see more or order reprints or permissions, see http://ijssurgery.com. 